\title{
A Fuzzy Control Heuristic Applied to Non-linear Dynamic System Using a Fuzzy Knowledge Representation
}

\author{
F.M. Cordova, G. Leyton
}

Felisa M. Cordova

University of Santiago of Chile

Ecuador 3769. Estacion Central

Chile, Santiago

E-mail: felisa.cordova@usach.cl

\section{Guillermo Leyton}

University of La Serena

Benavente 980

E-mail: gleyton@userena.cl

\begin{abstract}
This paper presents the design of a fuzzy control heuristic that can be applied for modeling nonlinear dynamic systems using a fuzzy knowledge representation. Nonlinear dynamic systems have been modeled traditionally on the basis of connections between the subsystems that compose it. Nevertheless, this model design does not consider some of the following problems: existing dynamics between the subsystems; order and priority of the connection between subsystems; degrees of influence or causality between subsystems; particular state of each subsystem and state of the system on the basis of the combination of the diverse states of the subsystems; positive or negative influences between subsystems. In this context, the main objective of this proposal is to manage the whole system state by managing the state combination of the subsystems involved. In the proposed design the diverse states of subsystems at different levels are represented by a knowledge base matrix of fuzzy intervals (KBMFI). This type of structure is a fuzzy hypercube that provides facilities operations like: insert, delete, and switching. It also allows Boolean operations between different KBMFI and inferences. Each subsystem in a specific level and its connectors are characterized by factors with fuzzy attributes represented by membership functions. Existing measures the degree of influence among the different levels are obtained (negatives, positives). In addition, the system state is determined based on the combination of the statements of the subsystems (stable, oscillatory, attractor, chaos). It allows introducing the dynamic effects in the calculation of each output level. The control and search of knowledge patterns are made by means of a fuzzy control heuristic. Finally, an application to the co-ordination of the activities among different levels of the operation of an underground mine is developed and discussed.

Keywords: Fuzzy Systems, Knowledge Representation, Heuristics, Nonlinear Dynamic Systems.
\end{abstract}

\section{Introduction}

Organizations can be visualized as complex systems composed of various subsystems that respond to different problems and have their own dynamics. This process in turn is recursive, so each subsystem has a particular dynamics. Such is the case of Managements, Business Areas, Departments, primary and support activities of the value chain, activities plans, besides many 
other systems and subsystems existing in the organization. Each subsystem is characterized by its variables and by inputs that can alter its performance and its outputs, which are the inputs of other subsystems, whose dependent effects are known only approximately. This constitutes a situation of a set of highly dynamic subsystems and with clearly nonlinear characteristics. Usually, these factors are not considered in the decision making processes.

It is clear that a universe of this kind is quite heterogeneous, dynamic, and growing. Also, because of the nature of the stated problem, it must be considered that these subsystems represent inputs among themselves, giving the problem a high dose of parallelism. Insofar as these subsystems serve as inputs among themselves, feedback is taking place continuously, making the system's dynamics difficult to control, predict, manage and administer [1]. It is also necessary to take into account the increasing number of data, information and knowledge that current systems must administer, in particular their adequate representation [9]. If we consider that the problem of knowledge-based management and decision making must be carried out in organizations having these characteristics, then it is ever more important to support conceptual models and tools adequate for the planning, management and control processes of this dynamics.

On the other hand, the representation knowledge is a fundamental component in any intelligent system that allows coding knowledge, objects, objectives, actions, and processes. The scheme for the chosen representation of knowledge determines the reasoning process and its efficiency. Numerous studies on the representation of knowledge show that a representation can be more adequate than another one for a particular case or it can be capable of covering a greater number of cases [8]. The more traditional methods used are Semantic Networks, Frames, Production Rules, Trees, and Bits Matrices. Cazorla et al. [3] suggest that knowledge can be classified according to the specific application to be used that develops knowledge: procedural, declarative, meta-knowledge, heuristic, or structural. However, the theory of diffuse sets proposed by Zadeh [12], [13] allows the generation of knowledge representations that are closer to the nature itself of what it is desired to represent.

The conceptual models of systems, their representation based on knowledge, and the tools for supporting management and decision making must then consider in their design factors such as high dynamism, parallelism, feedback, incompleteness, handling of uncertainty, nonlinearity, vagueness, qualitative definitions and behaviors, personal opinions, etc. Along this line, some authors [1], [16], [15] make a profound development of various concepts such as fuzzy function approximations, chaos and fuzzy control, and processing of fuzzy signals. However, his greatest contribution refers to the calculation and representation of knowledge by means of fuzzy cubes and fuzzy cognitive maps. McNeill [6] also works with fuzzy theory as a means of representing environments with uncertainty usually characterized by their nonlinearity. Welstead, on the other hand, supported by one of Kosko's results [11] suggests that fuzzy rules can be represented by one or more fuzzy associative memory matrices (FAM); combining the above with genetic algorithms he proposes a model to approach prediction problems. They also use fuzzy representations centered mainly on the interaction of fuzzy theory, neural networks, and genetic algorithms, supporting a new line of work known as Computational Intelligence. Tsoukalas [10] is more centered on the interaction and creation of fuzzy theory and neural network hybrids. To approach these kinds of problems, models are designed making use mainly of causal diagrams or knowledge maps with a series of nodes that would represent the concepts that are relevant to the system, and links between them that show the causal relation (influence) between concepts. In this context, the objective of this paper is to make a study and analysis that will allow modeling some types of dynamic systems, representing knowledge by means of a knowledge base matrix of fuzzy intervals and fuzzy cognitive maps [4], [14] and [15] with the purpose of achieving their categorization and fuzzy weight, as well as the levels of incidence in other subsystems, in this way characterizing the complete system with its levels of fuzzy incidence [5], [10]. 


\section{Modeling of the Diffuse Knowledge Base Matrix}

In this proposal each of the map's concepts corresponds to a fuzzy set, and it is specifically a particular Knowledge Base Matrix of Fuzzy Intervals (KBMFI). The connections between concepts will have an associated value in the $[-1,1]$ range that represents the degree of influence of a (KBMFI) node on another. If the value is positive, it indicates that an increase on the evidence of the origin concept increases the meaning, the evidence or the truth value of the destination concept. If it is negative, an increase of the evidence of the source causes a decrease of that of destination. If the value is 0 , there is no connection, and no causal relation.

In this way it is possible to get blurred cognitive maps from the opinion of one or various experts on the relations between some aspects of the evaluation process of a hypothetical case. Also, the clear recursiveness involved in these types of systems is considered, and a vision of granularity is proposed that allows overcoming the various levels of abstraction subjacent in the dissimilar subsystems. On the other hand, internally each subsystem can be represented by KBMFIs, allowing their incidence weight to be obtained with respect to other subsystems and at the same time represent their particular behavior.

Definition 1. Let $\mathrm{X}$ be a classical set of objects, called the universe. Belonging to a subset A of $\mathrm{X}$ can be defined in terms of the characteristic function:

$$
\mu_{A}: X \longrightarrow[0,1] x \longrightarrow \mu_{A}(x)
$$

where:

$$
\mu_{A}(x)= \begin{cases}1 & x \in A \\ 0 & x \notin A\end{cases}
$$

If the evaluation set 0,1 is extended to the real interval $[0,1]$, then it is possible to talk about the partial belonging in $A$, where $\mu_{A}(x)$ is the degree of belonging of $x$ in $A$, and the values 0 and 1 are interpreted as "non-belonging" and "total belonging", respectively.

Clearly, A is a subset of $\mathrm{x}$, which has no defined boundaries. This leads to the following definition.

Definition 2. Let X be an object's space. A fuzzy set A of X is characterized by the set of pairs:

$$
A=\left\{\left(x, \mu_{A}(x)\right) / x \in X\right\} \text { where } \mu_{A}: X \longrightarrow[0,1]
$$

The fuzzy concept proposed by Zadeh [11] is based on the fact of allowing the partial belonging in a set for certain elements of a given universe.

Definition 3. A fuzzy hypercube can be considered as a unit hypercube, i.e., a hypercube $\mathrm{I}^{\mathrm{n}}=[0,1]^{\mathrm{n}}$. The $\mathrm{n}$ fuzzy cube has two vertices or binary subsets.

A fuzzy cube contains all the fuzzy sets of a set X of $n$ objects. The non-fuzzy sets are found at the vertices of the cube. The continuum of fuzzy sets is in the cube.

Definition 4. Knowledge Base Matrix of Fuzzy Intervals (KBMFI) means the hypercube that is constituted by the various knowledge $E_{1}, E_{2}, E_{3}, \ldots, E_{n}$, relative to a domain of knowledge, considering also the different weight or importance that each of them has in the particular domain.

The KBMFI is a fuzzy hypercube where $E_{1}, E_{2}, \ldots, E_{n}$, represent the various contingencies or characteristics of the area under discussion, according to the opinion of the experts. $E_{j}$, with $j=1,2, \ldots, n$, do not necessarily have the same relevance or weight, they can be in particular 
fuzzy frames consisting of $S_{1}, \ldots, S_{m}$, where $S_{1}, S_{2}, \ldots, S_{m}$, are the possible factors, not necessarily disjoint, such that each characteristic $E_{i}$ can be expressed by means of some particular union of $S_{1}, S_{2}, \ldots, S_{m}$ factors. Now the particular determination of each $E_{i}$ through its particular factors $S_{1}, S_{2}, \ldots, S_{m}$, model systems composed of a range of nodes $N_{1}, N_{2}, \ldots, N_{n}$, continually influencing each other if and where the incidence of one with respect to others is completely dynamic. In particular, this outlines a vision of dynamic nonlinear systems which in similar but not equal versions are seen as causality maps.

If the map is adjusted to the opinions of several experts, one would have to get the assessments of all of them and therefore establish the definitive values associated with the causality relations. It must be noted that in general the causalities mentioned by the experts with respect to the various influences exerted by the nodes of the maps are more attributable to qualitative than quantitative concepts.

As already stated, nonlinear dynamic systems involve nonlinear and feedback behaviors. In these systems the output of a process or node is used as input for the following node or iteration, and the output of this can again be the input of the same previous node, i.e., self-recurrent behaviors. This behavior corresponds to the following equation:

$$
f\left(x_{0}\right)=\left\{\begin{array}{c}
X_{n-1} \\
X_{n} \\
X_{n+1}
\end{array}\right.
$$

Assuming that the following situation occurs when modeling the system: $x_{1}, x_{2}, x_{3}, \ldots, x_{n}$.

Definition 5. Let $x_{0}$ be an arbitrary starting node, then the above sequence is called the Trajectory.

Considering these definitions, several behaviors can occur, such as, for example: fixed points; periodic trajectories; behaviors given by attractor nodes, and chaos.

\section{Case Study}

The case study corresponds to the situation of an underground mine which has three levels: Production Level, Reduction Level, and Transport Level. The problem consists in "providing support to activity scheduling management". The problem consists in "providing support to activity scheduling management" [2]. The total system shown by Figure 1 consists of these three subsystems and the dynamics that exists between them. This situation is denoted as Level 1.

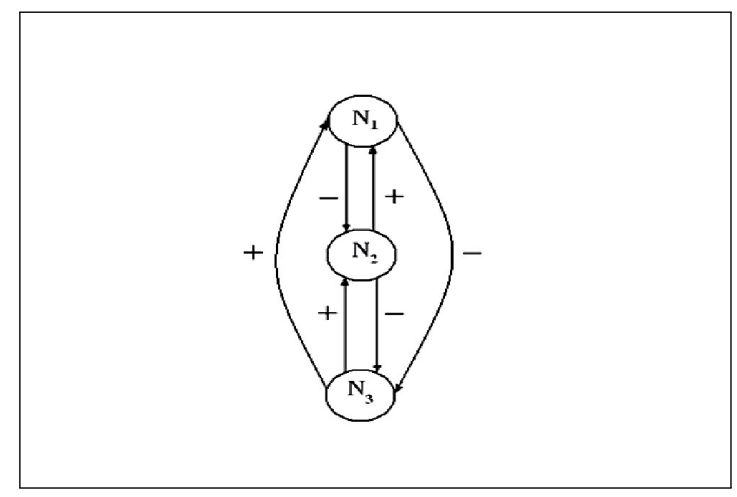

Figure 1: Production, Reduction and Transport Levels. 
$\mathrm{N}_{1}$ : Production Level considers $\mathrm{N}_{11}, \mathrm{~N}_{12}, \mathrm{~N}_{13}$, as subsystems; $\mathrm{N}_{2}$ : Reduction Level considers $\mathrm{N}_{21}, \mathrm{~N}_{22}$, as subsystems; $\mathrm{N}_{3}$ : Transport Level considers no subsystems.

Looking at it at a more particular abstraction level, Level 2 appears, as shown in Figure 2. From the particular situation shown, in Figure 1 it is seen that: $N_{1}$ influences $N_{2}$ negatively and $N_{3}$ positively, $N_{2}$ influences $N_{1}$ and $N_{3}$ positively, $N_{3}$ influences $N_{2}$ negatively and $N_{3}$ positively.

However, Figure 2 shows that the information obtained at Level 1 of abstraction of the system does not have the sensitivity or reliability that is obtained at Level 2 of abstraction, whose granularity or disaggregation is slightly higher.

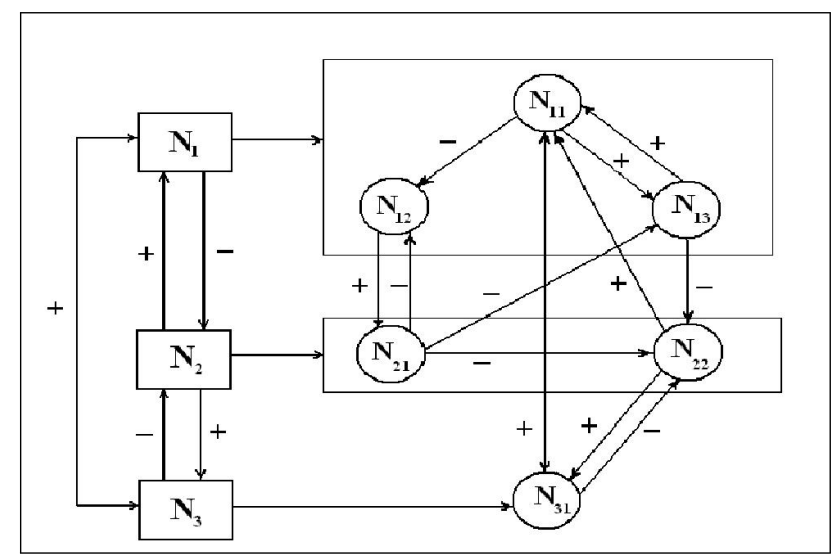

Figure 2: Diagram of influence at the different levels.

If both levels are confronted, it may be incorrectly deduced that apparently contradictory information is obtained. For example, if we look at Level 1 and Level 2 for the case of $\mathrm{N}_{3}$ with $\mathrm{N}_{2}$, at Level 1 it was stated that $\mathrm{N}_{3}$ influences $\mathrm{N}_{2}$ negatively, but at Level 2 it could be concluded that both have the opposite influence, $N_{31}$ influences $N_{22}$ negatively and $N_{21}$ influences $\mathrm{N}_{3} 1$ positively. This apparent contradiction can be explained, for example, by saying that when production at the Reduction Level decreases, there is less pressure on the demand for trains or cars, and on the other hand, if there is not sufficient transport from $\mathrm{N}_{31}$ there is an impact due to accumulation of material at the Reduction Level, which is considered a negative influence. Then the question is, which of the two situations has greater incidence weight? According to Figure 3, and only as an example, it can be stated that the negative impact from $N_{31}$ to $N_{22}$ is greater than the influence of $\mathrm{N}_{22}$ on $\mathrm{N}_{31}$.

The main observations to the system are: it is clear that it is a Dynamic Fuzzy System. In turn, every $N_{i}$ is a Dynamic Fuzzy Subsystem. The connections between the various $N_{i}$ are fuzzy. These connections can be positive or negative. If positive, $N_{i}$ influences positively on $N_{j}$. If negative, $\mathrm{N}_{i}$ influences negatively on $\mathrm{N}_{j}$.

\section{Design and Implementation of the KBMFI Matrix}

Going more deeply into Table 1, the experts draw these KBMFI as causal tables. They do not state equations, but make links between subsystems. The KBMFI systems convert each pictograph into a Fuzzy Rules Weight Matrix. The nodes of the KBMFI can model the complex nonlinearities between the input and output nodes. The KBMFI can model the dynamics that occur in the multiple iterations that take place in these dynamic systems.

The KBMFIs with $N$ nodes have $N^{n}$ arcs. Since $N_{i}(t)$ nodes are fuzzy concepts, their values $\in[0,1]$; a state of a KBMFI is the $\mathrm{N}_{\mathrm{i}}(\mathrm{t})=\left(\mathrm{N}_{1}(\mathrm{t}), \mathrm{N}_{2}(\mathrm{t}), \ldots, \mathrm{N}_{\mathrm{n}}(\mathrm{t})\right)$ vector, so it is a point of the 
hypercube $I^{\mathrm{n}}=[0,1]^{\mathrm{n}}$.

An inference in a KBMFI is a road or sequence of points in $\mathrm{I}^{\mathrm{n}}$, i.e., it is a fuzzy process or an indexed family of fuzzy sets $N(t)$. It is clearly seen that the KBMFIs can perform "forward chaining," and whether they can perform "backward chaining" (nonlinearity inverse causality) is an open question. The KBMFIs form, as nonlinear dynamic systems, Semantic Fuzzy Networks and act as neural networks. The KBMFIs can converge to a fixed point, to a limited cycle, that can be a stable or oscillating state or a chaotic attractor in the fuzzy cube In. In this context, one of the basic questions to be answered is: what happens if the input to the (KBMFI) system is known? In this sense, each KBMFI stores a set of global rules of the form:

\section{IF N(0) THEN attractor A}

A KBMFI with a single fixed global point has only one global rule. The size of the attractor regions in the fuzzy cube governs the number of these global regions or hidden patterns. The KBMFIs can have large and small attractor regions in In, each of them with a different degree of complexity. Therefore an input state can lead to chaos and a relatively close input state can end up in a fixed point or limited cycle or a stable state. Since the KBMFIs correspond to a Semantic Fuzzy Network structure, it is possible to associate a matrix M. This matrix lists the causal links between $N_{i}$ nodes. As an example, if it is considered again the case described by Figure 2, the corresponding KBMFI is presented where a row is the incidence of $N_{i}$ on $N_{j}$; columns are nodes influence $N_{i}$ and $\alpha, \beta, \gamma, \delta, \eta, \tau$, are values. Fuzzy function: [little, moreorless, much, etc.].

\begin{tabular}{c|cccccc} 
& $\mathrm{N}_{11}$ & $\mathrm{~N}_{12}$ & $\mathrm{~N}_{13}$ & $\mathrm{~N}_{21}$ & $\mathrm{~N}_{22}$ & $\mathrm{~N}_{31}$ \\
\hline $\mathrm{N}_{11}$ & 0 & $-\alpha \mu$ & $+\alpha \mu$ & 0 & 0 & $+\alpha \mu$ \\
$\mathrm{N}_{12}$ & 0 & 0 & 0 & $+\beta \mu$ & 0 & 0 \\
$\mathrm{~N}_{13}$ & $+\gamma \mu$ & 0 & 0 & 0 & $-\gamma \mu$ & 0 \\
$\mathrm{~N}_{21}$ & 0 & $-\eta \mu$ & $-\eta \mu$ & 0 & $-\eta \mu$ & 0 \\
$\mathrm{~N}_{22}$ & $+\delta \mu$ & 0 & 0 & 0 & 0 & $+\delta \mu$ \\
$\mathrm{N}_{31}$ & $+\tau \mu$ & 0 & 0 & 0 & $-\tau \mu$ & 0
\end{tabular}

The proposed model is decomposed in diverse abstraction levels and at each level is represented by a corresponding KBMFI. Initially, observing Figure 2, the Abstraction Level 0 appears. Only the influence shapes are observed. A Node $N_{i}$ can influence positively or negatively to the Node $\mathrm{N}_{\mathrm{j}}$. Abstraction Level 0 appears:

\begin{tabular}{c|ccc} 
& $\mathrm{N}_{1}$ & $\mathrm{~N}_{2}$ & $\mathrm{~N}_{3}$ \\
\hline $\mathrm{N}_{1}$ & 0 & - & + \\
$\mathrm{N}_{2}$ & + & 0 & - \\
$\mathrm{N}_{3}$ & + & - & 0
\end{tabular}

Experts are asked to qualify the degree of influence between: $\mu=$ [nothing, irrelevant, few, influence, regular, alter, a lot, very much, so much] as shown in the Incidence Graphic of Figure 3.

Applying the incidence graphic, a second level of abstraction 01 is obtained:

\begin{tabular}{c|ccc} 
& $\mathrm{N}_{1}$ & $\mathrm{~N}_{2}$ & $\mathrm{~N}_{3}$ \\
\hline $\mathrm{N}_{1}$ & 0 & $-\mu$ & $+\mu$ \\
$\mathrm{N}_{2}$ & $+\mu$ & 0 & $-\mu$ \\
$\mathrm{N}_{3}$ & $+\mu$ & $-\mu$ & 0
\end{tabular}

It is observed that the degree of incidence between a node $N_{i}$ with a node $N_{j}$, this means that it exists a bigger degree of specificity (granulation) between them. This enhancement of 


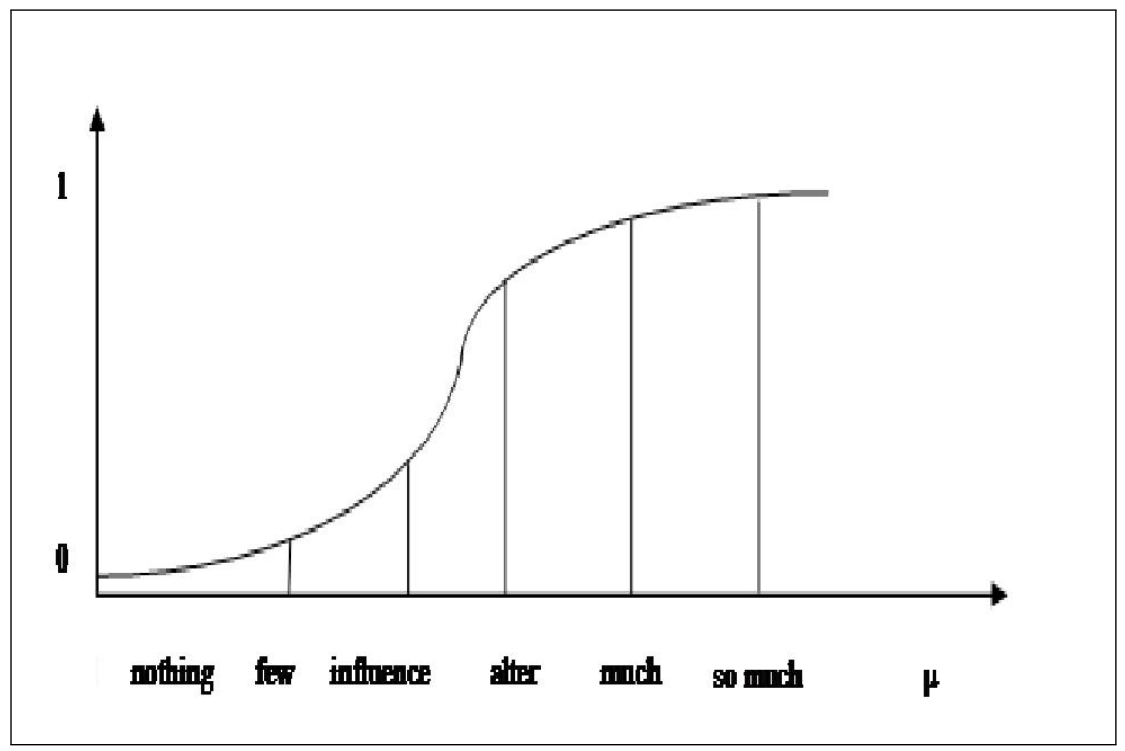

Figure 3: Incidence Graphic.

specificity is explicit in the following level, it exist a "slot" between $N_{i}$ with $N_{j}$. In this case different situations are denoted: $N_{1}$ influences in a negative way to $N_{2} ; N_{1}$ influences in a positive way to $N_{3} ; N_{2}$ influences in a positive way to $N_{1} ; N_{2}$ influences in a negative way to $N_{3} ; N_{3}$ influences in a positive way to $N_{1} ; N_{3}$ influences in a negative way to $N_{2}$.

If it is considered that a Node $N_{i}$ can be decomposed in $N_{i 1}, N_{i 2}, \ldots, N_{i k}$, in where those $N_{i m}$, $m=1,2, \ldots, k$, with a particular dynamic conforms a $N_{i}$, the situation in the analyzed case is as follows:

$N_{1}=\left(N_{11}, N_{12}, N_{13}\right)$; at Level 0; Node or Subsystem $N_{1}$.

$N_{1}$ at Level 01, Node or Subsystem $N_{1 i}$ is defined by:

\begin{tabular}{c|ccc} 
& $\mathrm{N}_{11}$ & $\mathrm{~N}_{12}$ & $\mathrm{~N}_{13}$ \\
\hline $\mathrm{N}_{11}$ & 0 & - & + \\
$\mathrm{N}_{12}$ & 0 & 0 & 0 \\
$\mathrm{~N}_{13}$ & + & 0 & 0
\end{tabular}

$N_{1}$ at Level 011 is defined by:

\begin{tabular}{c|ccc} 
& $\mathrm{N}_{11}$ & $\mathrm{~N}_{12}$ & $\mathrm{~N}_{13}$ \\
\hline $\mathrm{N}_{11}$ & 0 & $-\alpha \mu$ & $+\alpha \mu$ \\
$\mathrm{N}_{12}$ & 0 & 0 & 0 \\
$\mathrm{~N}_{13}$ & $+\alpha \mu$ & 0 & 0
\end{tabular}

$\mathrm{N} 2=\mathrm{N}_{21}, \mathrm{~N}_{22}$; at Level 0 ; Node or Subsystem $\mathrm{N}_{2}$.

$N_{2}$ at Level 01, Node or Subsystem $N_{2 i}$ is defined by:

\begin{tabular}{c|cc} 
& $\mathrm{N}_{21}$ & $\mathrm{~N}_{22}$ \\
\hline $\mathrm{N}_{21}$ & 0 & - \\
$\mathrm{N}_{22}$ & 0 & 0
\end{tabular}

$N_{2}$ at Level 011 is defined by: 


\begin{tabular}{c|cc} 
& $\mathrm{N}_{21}$ & $\mathrm{~N}_{22}$ \\
\hline $\mathrm{N}_{21}$ & 0 & $-\eta \mu$ \\
$\mathrm{N}_{22}$ & 0 & 0
\end{tabular}

Applying the same procedure to node $\mathrm{N}_{3}$ and it is only characterized by $\mathrm{N}_{31}, \mathrm{~N}_{3}$ at Level 011 is defined by:

$$
\begin{array}{c|c} 
& \mathrm{N}_{31} \\
\hline \mathrm{N}_{31} & 0
\end{array}
$$

At this point only the fuzzy subsystem cohesion is developed. So, it is necessary to visualize what it happens with the external dynamic between subsystems, in order to obtain the fuzzy matching inter systems. Continuing with the fuzzy cohesion procedure, links between nodes $\mathrm{N}_{1}$, $\mathrm{N}_{2}$ and $\mathrm{N}_{3}$, at Level 0 by Nodes are obtained:

\begin{tabular}{c|ccc} 
& $\mathrm{N}_{1}$ & $\mathrm{~N}_{2}$ & $\mathrm{~N}_{3}$ \\
\hline $\mathrm{N}_{1}$ & 0 & - & +
\end{tabular}

At Level 01 by Node $N_{1}$ :

\begin{tabular}{c|ccc} 
& $\mathrm{N}_{1}$ & $\mathrm{~N}_{2}$ & $\mathrm{~N}_{3}$ \\
\hline $\mathrm{N}_{1}$ & 0 & $-\alpha \mu$ & $+\alpha \mu$
\end{tabular}

At Level 011 by Node $N_{1}$ :

\begin{tabular}{c|cccc} 
& $\mathrm{N}_{1}$ & $\mathrm{~N}_{21}$ & $\mathrm{~N}_{22}$ & $\mathrm{~N}_{31}$ \\
\hline $\mathrm{N}_{11}$ & 0 & 0 & 0 & $+\alpha \mu$
\end{tabular}

At Level 02 by Node $N_{1}$ :

\begin{tabular}{c|cccc} 
& $\mathrm{N}_{1}$ & $\mathrm{~N}_{21}$ & $\mathrm{~N}_{22}$ & $\mathrm{~N}_{31}$ \\
\hline $\mathrm{N}_{11}$ & 0 & $+\beta \mu$ & 0 & 0
\end{tabular}

In this way, influences are obtained allowing the fuzzy matching.

\section{Heuristic Control for the KBMFI}

Each $N_{i}$ level has $F_{i j}$ factors that determine it, with $i=1,2,3 ; j=1,2, \ldots, m$. Table 1 shows relevant characteristics, factors, attributes and fuzzy functions at Production Level.

Table 2 shows relevant factors, attributes and fuzzy functions at Production Level.

Each $F_{i j}$ factor has $A_{i j} s$ attributes that determine it, where $i=1,2,3 ; j=1,2, \ldots, m ; s=$ $1,2, \ldots, k$ (see Table 1$)$.

Each $A_{i j}$ s has attribute metrics associated with its nature. These metrics are functions of fuzzy membership (see Table 2).

For the above points, it is possible to state that the degrees of influence (negative or positive) that exist between the various levels can be measured, allowing the calculation of the existing dynamics of the system to achieve an Intelligent Fuzzy Control with the purpose of keeping the system in a desirable state (stable). 


\begin{tabular}{|l|l|l|l|l|l|l|l|l|l|l|}
\hline $\begin{array}{l}\text { CHARACTERISTICS OF LEVEL } \\
1 \text { (PRODUCTION) }\end{array}$ & ATTRIBUTES (Metrics or fuzzy functions \\
\hline & Table 1.1) & & \multicolumn{7}{|c|}{ Fuzzy Functions } \\
\hline \multicolumn{1}{|c|}{ FACTORS 1 } & Rel. Card. (CRC) & 1 & 2 & 3 & 4 & 5 & 6 & 7 & 8 & 9 \\
\hline 1. Number of workmen present & & & & & & & & & & \\
\hline 2. Drilling, agents, and resources & & & & & & & & & & \\
\hline 3. Blasting, agents and resources & & & & & & & & & & \\
\hline 4. Technologies involved & & & & & & & & & & \\
\hline 5. Number of equipments & & & & & & & & \\
\hline 6. Lectures & & & & & & & \\
\hline \\
Relative cardinality of Level 1 (CRN1)
\end{tabular}

Table 1: Relevant characteristics of Level 1 at Production Level.

\section{Heuristic}

The proposed heuristic consists of the following stages:

Stage 1: Obtaining the $F_{i j}$ factors of each level $N_{i}$.

Stage 2: Obtaining the $A_{i j} s$ attributes of each $F_{i j}$ factor.

Stage 3: Determining the metrics associated with each $A_{i j} s$ attribute, i.e., determining the fuzzy membership functions for each $A_{i j} s$.

Stage 4: Determining the "formula" that corresponds to each $F_{i j}$ from the $A_{i j} s$, for example:

$$
F_{i j}=\lambda_{1} A_{i j} 1 \oplus \lambda_{2} A_{i j} 2 \oplus \ldots \oplus \lambda_{k} A_{i j} k
$$

where $\oplus$ is the operator to be determined $(=>, \vee, \cup$, etc. $)$ and $\sum \lambda_{k}=1$.

Stage 5: Determining $N_{i}$ from the $F_{i j}$, for example:

$$
\mathrm{N}_{i}=\lambda_{1} \mathrm{~F}_{\mathrm{i} 1} \oplus \lambda_{2} \mathrm{~F}_{\mathrm{i} 2} \oplus \ldots \text { oplus } \lambda_{\mathrm{m}} \mathrm{F}_{\mathrm{im}}
$$

where $\oplus$ is the operator to be determined $(=>, \vee, \cup$, etc. $)$ and $\sum \lambda_{\mathrm{m}}=1$. Note that the output of all $\mathrm{N}_{j}$ must be between 0 and 1 .

Stage 6: Determining whether the "influence" of the output of $N_{i}$ to other levels is negative or positive.

Stage 7: Recalculating the $\mathrm{N}_{\mathrm{t}}$ output, with its internal values, considering the influence exerted on it by the recursive dynamics of the nodes $\mathrm{N}_{i}$ at Stages $1,2, \ldots, 5$.

Stage 8: Determining the output of $N_{t}$, input of $N_{l}$, and determining whether we feed $N_{l}$ or $\mathrm{N}_{i}$, and specifying the times. Note that in this step we distinguish between what influences what, or we make a push, we make a pull, or both at the same time, with a delay of one with respect to the other, etc. 


\begin{tabular}{|c|c|}
\hline FACTORS AND ATTRIBUTES & FUZZY FUNCTIONS \\
\hline PROJECT SYSTEM & \\
\hline 1. Number of workmen present. & \\
\hline (Decision making complexity). & \\
\hline 1.1 Number of engineers. & $\mu_{1}^{1}(x)=1-\frac{25-x}{25} \quad 10 \leq x \leq 25$ \\
\hline 1.2 Number of technicians. & $\mu_{2}^{1}(x)=1-\left(\frac{75-x}{75}\right)^{2} \quad 30 \leq x \leq 75$ \\
\hline 1.3 Number of miners. & $\mu_{3}^{1}(x)=1-\left(\frac{150-x}{150}\right)^{2}$ \\
\hline 1.4 Number of equipments & $\begin{array}{l}\mu_{4}^{1}(x)=1-\frac{30-x}{30} \quad 12 \leq x \leq 30 \\
\chi=\text { amount of engineers, miners, ... }\end{array}$ \\
\hline 2. Drilling, agents and resources. & $\begin{array}{l}\text { For evaluating this characteristic, first the predominant fac- } \\
\text { tor must be identified and then the calculation can be made. } \\
\text { For example, if } x=25 \text { or } 30 \text { or } 90 \text { or } 21 \text {, for respective: } \\
\mu_{i}^{1}: \mu_{1}^{1}(25)=1 ; \mu_{2}^{1}(30)=0.64 ; \mu_{3}^{1}(90)=0.84 ; \mu_{4}^{1}(21)=0.91\end{array}$ \\
\hline 2.2 Direct agents involved. & $\mu_{1}^{2}(x)=1-\sqrt{\frac{50-x}{50}} \quad 20 \leq x \leq 50$ \\
\hline 2.3 Indirect agents involved. & $\mu_{2,3}^{2}(x)=1-\left(\frac{15-x}{30}\right)^{3} \quad 6 \leq x \leq 15$ \\
\hline
\end{tabular}

Table 2: Factors, attributes and fuzzy functions at Production Level.

\section{Conclusions and Future Works}

The work done in the paper allows the characterization of a complex system through subsystems considering the dynamics and the incidence of each subsystem on the others. From the display of the complexity of the system and subsystems, the KBMFI is constructed, which allows an adequate representation of diffuse knowledge and the dynamics associated with the system. A fuzzy control heuristic is also designed that allows managing the KBMFI.

In the case of the planning of mining operations, the KBMFI and the associated heuristic allow the evaluation of the impact of the incidence of various factors such as reduction of the number of planned workers in a shift, faults in Load Haul and Dump LHD equipment, rock breakers, shafts, and trains, among others.

If someone is considering developing software from this proposal, it should be kept in mind that in the tool there should be an agent module that is informed (alert) of the acceptable critical values for each node, so that this node does not alter acceptable states (experts) of the nodes with which it interacts. In such case the agent must learn about the acceptable critical values, know and learn preventive measures; know and learn mitigation measures, and know and learn corrective measures.

\section{Bibliography}

[1] D. Alahakoon, S. K. Halgamuge, and B. Srinivasan, Dynamic self organizing maps with controlled growth for knowledge discovery, IEEE Trans. Neural Networks, Vol.11:601-614, 2000 .

[2] F. Cordova, L. Canete, L. Quezada, F. Yanine, An Intelligent Supervising System for the Operation of an Undergound Mine, International Journal of Computers, Communications and Control, Vol. III: 259-269, 2008.

[3] M. Gupta, R.K. Ragade,Yager, Advances in Fuzzy Sets Theory and applications, North Holland, Amsterdam, 1979. 
[4] B. Kosko, Fuzzy Ingineering. Prentice Hall, 1997.

[5] G. Martinez, Servente and Pasquini,Sistemas Inteligentes, NL Nueva Libreria, Argentina, 2003.

[6] T. McNeill, Fuzzy Logic a Practical Approach. Academic Press, 1997.

[7] H. Roman, Sobre Entropias Fuzzy, Tesis de doctorado, Universidad de Campinas, Brasil, 1989.

[8] E. Schnaider, A. Kandel, Applications of the Negation Operator in Fuzzy Production Rules, Fuzzy Sets and Systems, Vol. 34: 293-299, Noth Holland, 1990.

[9] W. Silder, J. Buckley, Fuzzy expert system and fuzzy reasoning, John Wiley and Sons Inc., New Jersey, 416, 2005.

[10] U. Tsoukalas, Fuzzy and Neural Approaches in Engineering. Wiley Interscience, 1997.

[11] S. Welstead, Neural Network and Fuzzy Logic Applications in $\mathrm{C}++$. Wiley Interscience, 1994.

[12] L.A. Zadeh, The role of fuzzy logic in the management of uncertainly in Expert Systems, Aproximate Reasoning in Expert Systems, Elservier Science Pub., North Holland, 3-31, 1985.

[13] L.A. Zadeh et al (eds.), From Natural Language to Soft Computing: New Paradigms in Artificial Intelligence, Editing House of Romanian Academy, 2008.

[14] Zadeh, L.A., Outline of a new approach to the analysis of a complex systems and decision processed, IEEE Trans. Syst. Man Cybern., Vol. 3: 28-44, 1973.

[15] L.A. Zadeh, Fuzzy sets and fuzzy information: granulation theory, Beijing Normal University Press, Beijing, 1997.

[16] L.Zhong, W.A. Halang,G. Chen, Integration of Fuzzy Logic and Chaos Theory, SpringerVerlag, Berlin Heidelberg, 2006. 